\title{
Development of a fall prevention protocol for replication in a virtual environment
}

\author{
Beth Ann Walker ${ }^{1 *}$, Lesa L. Huber ${ }^{2}$ \\ ${ }^{1}$ School of Occupational Therapy, University of Indianapolis, Indianapolis, USA; \\ *Corresponding Author: walkerba@uindy.edu \\ ${ }^{2}$ School of Health, Physical Education, and Recreation, Indiana University, Bloomington, USA; \\ lehuber@indiana.edu
}

Received 4 July 2012; revised 8 August 2012; accepted 16 August 2012

\begin{abstract}
Several effective fall prevention exercise programs address the problem of falls. The primary challenge is not to develop effective programs, but to find ways to motivate older people to begin and maintain these programs while delivering these programs to as many older adults as possible. This short-term pilot study collected preliminary data on the design and testing of a balance rehabilitation protocol that could be replicated in a virtual gaming environment like the Wii Fit. The protocol, based on evidencebased practice, focused on strength, endurance, and functional reach. Eight older adults participated in twelve one hour sessions with an occupational therapist and exercise professional. Outcome measures included the Modified Falls Efficacy Scale, Life Space Questionnaire, and postural sway. Results suggest that the protocol was effective in improving postural sway (as measured by a force plate), reducing fear of falling, and increasing perceived life space.
\end{abstract}

Keywords: Fall Prevention; Virtual Gaming; Exercise; Older Adults; Balance

\section{INTRODUCTION}

The majority of older adults want to be able to live and function independently. People want to exercise control over their lives and continue to live at home as long as possible. However, a fall can have a significant impact on the ability to function independently. Falls often lead to serious injury, including hip fractures and head injuries. Hospitalization and immobilization after a fall often triggers a series of cascading disabilities. Many older adults who have had a serious fall never return home. What we don't know about fall rates is sobering: many older adults do not report falls, fearing institutionalization and loss of personal autonomy based on their actual rate of falling. As a result, many frail older adults, particularly those that live alone, risk falling and not being able to summon help.

According to the Centers for Disease Control and Prevention (CDC), approximately one-third of older adults, aged 65 and older, fall each year [1-3]. It is also estimated that twenty to thirty percent of those individuals that fall suffer from moderate to severe injuries that greatly impact their ability to complete activities of daily living $[1,4]$. Perhaps most importantly, falling in the elderly produces negative consequences that actually increase a person's risk for falling-over $70 \%$ of elderly subjects who have experienced a fall acknowledge avoiding previously performed activities such as walking, bathing or housekeeping because of this fear of falling [5].

Falls not only impact the quality of life of older adults but also increase the financial burden on the healthcare systems of our country. The CDC reported that in 2000, the total direct cost of all fall injuries for older adults aged 65 and older exceeded $\$ 19$ billion [6]. Adding to the complexity of the issue is the fact that the population of older Americans is rising at an unprecedented rate. According to the U.S. Census Bureau [7], all of the baby boomers will be 65 and older in 2030 and this population is expected to grow to approximately 88.5 million by 2050 . It can be anticipated that approximately 29.5 million older American adults will fall in 2050. Thus, it will be imperative to find real life health solutions to issues that will impact a large segment of our population. Related costs from permanent disability, institutionalization and even death are growing as the older adult population continues to grow [1].

Another concern is the fact that as America ages, eventually there will not be a sufficient number of young adults to provide services for senior citizens, especially in the realm of fall prevention through physical activity and functional rehabilitation. Rehabilitation professionals are already under the economic pressure of current health-care 
trends and are pressed to provide quality services to individuals that spend less time in the hospital and have less therapy allotted to them. There is a critical need for rehabilitation modalities that are low-cost, of high-quality and able to ensure carry-over of success without relying on the physical presence of a rehabilitation practitioner.

Virtual gaming interventions may provide an opportunity to provide classes for older adults that find it challenging to access and participate in real-person, group falls intervention activities. The Wii Fit has the potential to address common barriers to exercise: availability of well-designed interventions, cost, transportation, boredom/discomfort, and preference for home-based interventions. The gaming platform also allows remote observation of user performance, obtains objective data, and provides real-time feedback. However, the existing Wii Fit platform was not designed to improve balance and reduce fear of falling in older adults.

Several studies have identified modifiable risk factors of falls among older adults including muscle weakness, gait and balance problems, poor vision, urinary incontinence, use of psychoactive medications and structural hazards within the home [6]. The Compendium of Effective Community-Based Interventions [1] identified six exercise-based fall prevention programs that have rigorous scientific evidence of effectiveness: Stay Safe, Stay Active, the Otago Exercise Program, Tai Chi: Moving for Better Balance, Australian Group Exercise Program, Veterans Affairs Group Exercise Program, and Simplified Tai Chi.

While these programs are shown to be effective, they are not available, accessible, or enjoyable for all older adults. Adding to this conundrum is that studies have estimated that between $1 / 3$ and $1 / 2$ of Americans over 50 get no physical activity at all, and the number is even higher for women, minorities, and the very old [8]. Thus, it is not surprising that fall prevention efforts often fail due to low levels of motivation, participation, adherence, self-efficacy, and accountability. Therefore, the primary challenge is not only to develop a fall prevention program that effectively reduce the risk of falling, but to find ways to motivate older people to begin and maintain an effective fall prevention programs, as well as, encourage carry-over of success beyond program end.

Arguably the most popular physical fitness video game, the Wii Plus, was designed to operate as a virtual coach, and guide users through various basic strength, balance, and aerobic activities to improve both physical fitness and coordination. The gaming mechanisms have also been shown to allow remote observation of user performance, obtain objective data, increase motivation, distract from discomfort, and provide real-time feedback to allow people to better track their progress [9]. The Wii Fit Plus also addresses the barriers to participation in exercise that older adults face: availability of well-designed interventions, cost, transportation to community based programs, racial/ethnic barriers to participation in community-based programs, boredom/discomfort with standard exercise, and preference for the convenience of homebased interventions.

There are numerous games that can be played on the current Wii Fit that improve strength, cardiorespiratory capacity, and balance. Video games have been shown to allow remote observation of user performance, obtain objective data, increase motivation, distract from discomfort, and provide real-time feedback to allow people to better track their progress [9]. Many of the activities, however, have fast-paced, moving graphics that older adults find distracting or are not age appropriate. There are limited options in exercise progressions.

From a rehabilitation perspective, research suggests that video games do appear to have potential for improving older adults' physical and cognitive health, increasing enjoyment during exercise and even improving postural sway. Basak, Boot, Voss, and Kramer [10] found that significant benefits were realized following strategy-based video game training on executive control functions. In a review of studies, Green and Bavalier [11] found that video game play can improve perceptual, motor, and cognitive function in older persons. Van Schaik, Blake, Pernet, Spears, \& Fencott, [12] suggest that a cognitive exercise such as solving simple puzzles during physical exercise can break a negative link between perception of time spent exercising and performance by providing a distraction from the physical exercise. Elder participants strongly preferred virtual augmented exercise to traditional physical exercise, and adherence rate was $100 \%$. Haddad, Van Emmerik, Wheat, and Hamill [13] found that postural sway is especially challenged during supra-postural tasks such as a manual fitting task, and can reveal age-related declines in posture and balance in individuals as young as 40 years of age. As a result, improving functional reaching of older adults through supra-postural tasks has the potential to improve their balance.

The purpose of this pilot study was to assess the feasibility and effectiveness of a hybrid fall prevention rehabilitation exercise program. The hybrid program focused on fundamental components of the evidence-based FallProof program [14] and was designed to be replicated in a virtual environment. It was hypothesized this hybrid fall prevention intervention would improve balance, fear of falling, and life space mobility, as defined by outcome measures.

\section{METHODS}

\subsection{Study Design}

This was an exploratory pilot study using a pre- and 
post-test design. This pilot study also offered an opportunity to explore issues of feasibility. This project was approved by the Indiana University Bloomington Institutional Review Board.

\subsection{Participants}

Eight community-dwelling older adults aged 68 years and above participated in a repeated measures ABA (baseline-intervention-baseline) pilot study. Inclusion criteria were: older adults aged 65 - 90 years old living in the community and not currently enrolled in a formal exercise class. Exclusion criteria were moderate to severe cognitive problems (score $<31$ on the Telephone Interview for Cognitive Status [15] and musculoskeletal or neurological comorbidity that would preclude the planned exercises or result in motor performance difficulties.

\subsection{Instrumentation}

The Life Space Questionnaire (LSQ) was used to measure the extent of participant's community mobility as measured by the distance that the participant travels within their home, neighborhood, and outside their community [16]. The LSQ consists of 9 yes/no interview questions related to the participant's mobility over the previous 3 days. Example items include "During the past 3 days, have you been to an area outside your home such as your porch, deck, or patio, hallway of an apartment building, garage?" and "During the past 3 days, have you been to places outside your immediate town or community"? Items are scored either 1 ("yes") or 2 ("no") and only the yes's are summed (range $=1-9$ ).

Fear of falling was measured using the Modified Falls Efficacy Scale (MFES). The MFES aims to determine how confident seniors feel they are able to undertake various activities without being afraid of falling [17]. Example items include "Get dressed and undressed" and "Using front or rear steps at home." Items are scored from 0 ("not confident at all") to 10 ("completely confident"). Responses are summed (range $=0$ - 140).

Postural sway was measured by having participants stand on an AMTI forceplate for 30 seconds to assess their displacement of center of pressure (COP). The AMTInetforce software program was used for the data recording and the exported data was analyzed using Matlabb. The parameter measured was the sway area.

Demographic data was collected from each participant using the FallProof Health and Activity Questionnaire which includes information such as birth date, age, sex, height, weight, home environment, social and family history, medical history, ambulation, assistive device use, medications, participation in activities, current concern for falling, past falls before the intervention, and activities of daily living [14].

\subsection{Intervention}

The hybrid fall prevention exercise program was developed through a user-centered, iterative design process with the authors and Advisory Board. Advisory Board members included faculty in Kinesiology and Informatics and fitness specialists with experience in teaching older adults and balance. This collaborative interdisciplinary team reviewed evidence-based exercise protocols for their ability to improve balance, reduce fear of falls, and ability to be replicated in an enjoyable gaming environment. A focus on functional training education was incorporated through specific exercises which can be applied to daily living activities [18]. Understanding sense of purpose for exercise is critical for exercise adherence to make a difference in balance and falls.

The hybrid fall prevention rehabilitation exercise protocol was an integration of the FallProof comprehensive balance and mobility training program [14] and functional training education. Specific exercises were chosen based on their translational ability to a virtual environment. The protocol was composed of the following components: center of gravity control, multisensory training, postural strategy training, gait pattern enhancement and variation training, strength and endurance training, and flexibility training.

Center of gravity control training exercises included exercises such as seated balance activities and standing balance activities that involve reaching and leaning. Multisensory training exercises included activities that focus on eye-head coordination and exercises such as standing while focusing on an object. Postural strategy training included activities that incorporate leaning while standing and stepping sideways or backwards. Gait pattern enhancement and variation training included taking steps with directional changes or simulated walking a tight rope. Strength and endurance training included standard upper and lower extremity exercises such as leg lifts while seated and bicep curls. Flexibility training included exercises such as seated head/neck stretches, and full-body stretches [14].

Participants participated in the hybrid exercise protocol 2 times per week for six weeks. Each session lasted for 60 minutes. Four participants met each week in the exercise room of their retirement community. The other participants were seen in their own homes.

\subsection{Data Analysis}

For each outcome measure descriptive analyses including mean and standard deviations were generated. Effect sizes were calculated using the equation: ([meanpost meanpre]/SDpooled) [19]. Effect size for each outcome measure was reported. An effect size of 0.2 was considered small, 0.5 was considered medium and 0.8 was con- 
sidered large [20].

\section{RESULTS}

Table 1 summarizes the demographics of the 8 participants. The average age of the participants was 76 . Seven of the eight participants were female and all were white/ non-Hispanic. All had TICS scores that indicated normal cognitive status. One participant used an assistive device.

There was $100 \%$ participation; all 8 participants recruited completed the 6 week hybrid fall prevention rehabilitation exercise program, pretesting, and posttesting. Posttest scores were casewise deleted for one participant because of incomplete items. Casewise deletion was chosen to be the best method for handling this missing data as the participant was experiencing illness unrelated to the intervention on the day of posttesting [21].

\section{Outcomes}

Outcome data are reported for 7 of the 8 individuals that participated in the study. Results of outcome measures and overall mean scores can be found in Table 2 . Effect sizes for all outcome measures are estimated in Table 3. Overall mean scores increased for the LSQ. LSQ scores pre-intervention and post-intervention were 5.57 and 6.57, respectively. Participants had similar MFES scores pre and post intervention with overall mean scores 9.32 and 9.4 respectively. Three participants (43\%) experienced no change in their fear of falling after the completion of the intervention. Two participants' scores indicated a change from essentially not being worried at all to being a little worried about falls following the intervention. Two participants' scores indicated a reduction in fear of falling after the intervention. Overall mean postural sway area decreased from an average of $380.11 \mathrm{~mm}$ 2 to $263.0 \mathrm{~mm} 2$.

\section{DISCUSSION}

The purpose of this initial study is to collect preliminary data on the design and testing of a rehabilitation protocol focused on strength, endurance, and functional reach that improved postural sway and reduces fear of

Table 1. Participant characteristics.

\begin{tabular}{ccccccccc}
\hline & \multicolumn{8}{c}{ Participants } \\
\hline Age & 1 & 2 & 3 & 4 & 5 & 6 & 7 & 8 \\
Sex & F & F & F & M & F & F & F & F \\
Race & W & W & W & W & W & W & W & W \\
TICS & 33 & 44 & 37 & 36 & 32 & 41 & 45 & 33 \\
Assistive Device & & RW & & & & & & Cane \\
\hline
\end{tabular}

Table 2. Outcome measure results.

\begin{tabular}{ccccccc}
\hline Participant & \multicolumn{2}{c}{ LSQ } & \multicolumn{2}{c}{ MFES } & \multicolumn{2}{c}{ Sway } \\
\hline & Pre & Post & Pre & Post & Pre & Post \\
\hline 1 & 7 & 7 & 9.57 & 10 & 24.66 & 34.46 \\
2 & 2 & 5 & 5.79 & 7.29 & 226.27 & 162.18 \\
3 & 5 & 7 & 10 & 10 & 171.3 & 101.56 \\
4 & 7 & 7 & 10 & 10 & 811.88 & 359.7 \\
5 & 6 & 6 & 10 & 10 & 713.25 & 569.27 \\
6 & 7 & 5 & 9.86 & 8.78 & 392.24 & 354.27 \\
7 & 5 & 9 & 10 & 9.75 & 321.18 & 259.58 \\
Avg & 5.57 & 6.57 & 9.32 & 9.40 & 380.11 & 263.00 \\
SD & 1.81 & 1.4 & 1.56 & 1.03 & 287.12 & 182.55 \\
\hline
\end{tabular}

Table 3. Effect size estimations.

\begin{tabular}{cc}
\hline Outcome measures & Effect size cohen's $d^{*}$ \\
\hline MFES & 0.06 \\
LSQ & 0.62 \\
Sway & 0.49 \\
\hline
\end{tabular}

*Effect sizes: small $=0.2 ;$ medium $=0.5 ;$ large $=0.8$.

falling. A hybrid of exercises was selected based on their ability to be replicated in a virtual gaming environment like the Wii Fit. This study adds to the body of literature exploring the feasibility of creating rehabilitation quality gaming specifically designed for the functional needs of older adults and derived from evidence-based practice.

A user-centered, iterative design process was used to create a hybrid fall prevention rehabilitation exercise protocol. The protocol was based on the following components of the FallProof [14] comprehensive balance and mobility training program: center of gravity control, multisensory training, postural strategy training, gait pattern enhancement and variation training, strength and endurance training, and flexibility training. The protocol was reviewed and amended by an interdisciplinary panel of experts.

In this study, participants' perceived life space increased after the intervention. These results suggest that the hybrid protocol was effective in helping participants maintain or increase activities outside the home, neighborhood, and community. Fear of falling was also reduced after the intervention, as measured using the Modified Falls Efficacy Scale (MFES). One participant stated "I went to a garage sale next door yesterday, and I haven't even attempted to go to my mailbox in a long time for fear of falling". This suggests that the hybrid protocol can be effective in increasing confidence about balance and decreasing the threats to socialization, independence and morbidity/mortality [22].

The effect size was not large, but with such a small sample, a limited case analysis is somewhat helpful in pro- 
viding insights into the results. Outcome data had to be deleted for one participant due to missing items. This participant had a sudden change in health status unrelated to the intervention which may have affected her ability to complete the instruments. This participant however was severely deconditioned at the beginning of the intervention and had probably made the most improvements in balance, fear of falling, and life space.

Participant 6 also showed a decline in LS and MFES assessments. Qualitative field comments with this participant indicated that she had recently changed some medications due to an injury she sustained prior to the intervention. This recent change affected her perceptions on the LS and MFES and explained the decline. Her sway scores showed improvement pre/posttest. She noted that she felt her balance had improved during the intervention, had declined since the intervention ended, and the exercises were "amazing."

Finally, pretest scores on the LS and MFES were rather high for all participants. As the intervention began, it became obvious that not all participants were as balance-proficient as their pretests would indicate. Because falling can be a reason for institutionalization, older adults sometimes do not report or under report falls and fear of falling [22]. It is possible that participants exhibited a Hawthorne effect in their pre test scores. Consequently, their post test scores reflected a more realistic assessment resulting from increased familiarity and comfort with the instructors and authors. Effect sizes may have been larger if pretest scores on the LS and MFES had been more realistically aligned with actual balance abilities.

This study confirms that virtual gaming interventions may provide an opportunity to provide classes for older adults that find it challenging to access and participate in real-person, group falls intervention activities. One participant stated "I will try anything if it means I might not fall so much". The gaming platform also appears to distract from discomfort while also providing a source of active entertainment. One participant stated "this reminds me of doing the fox trot... it takes me back to when my body was fit and able. Boy could I dance".

\section{CONCLUSION}

The primary goal of this pilot study was to explore the development and feasibility of a rehabilitation based fall prevention protocol to be replicated in a virtual gaming environment. Findings indicate that the protocol does have potential. The study design was sufficient for our purpose. In the future, a larger randomized trial will be needed to determine whether a virtual gaming rehab-based fall prevention program would be effective. Future studies also need to broaden outcomes to include participation, carryover, and remote observation.

\section{REFERENCES}

[1] Center for Disease Control (2008) Preventing falls: What works. A compendium of effective community-based interventions from around the world. CDC, Atlanta.

[2] Hornbrook, M., Stevens, V., Wingfield, D., Hollis, J., Greenlick, M. and Ory, M. (1994) Preventing falls among community-dwelling older persons: Results from a randomized trial. The Gerontologist, 34, 16-23.

doi:10.1093/geront/34.1.16

[3] Hausdorff, J., Rios, D. and Edelber, H. (2001) Gait variability and fall risk in community-living older adults: A 1-year prospective study. Archives of Physical Medicine and Rehabilitation, 82, 1050-1056. doi:10.1053/apmr.2001.24893

[4] Alexander, B., Rivara, F. and Wolf, M. (1992) The cost and frequency of hospitalization for fall-related injuries in older adults. American Journal of Public Health, 82, 1020-1023. doi:10.2105/AJPH.82.7.1020

[5] Tinetti, M.E., Baker, D.I., McAvay, G., Claus, E.B., Garrett, P., Gottschalk, M., et al. (1994) A multifactorial intervention to reduce the risk of falling among elderly people living in the community. New England Journal of Medicine, 331, 821-827. doi:10.1056/NEJM199409293311301

[6] Stevens, J., Corso, P., Finkelstein, E. and Miller, T., (2006) The costs of fatal and nonfatal falls among older adults. Injury Prevention, 12, 290-295. doi:10.1136/ip.2005.011015

[7] US Census Bureau (2008) An older and more diverse nation by midcentury.

http://www.census.gov/Press-Release/www/releases/archi ves/population/012496.html

[8] Chodzko-Zajko, W. (2006) National Blueprint: Increasing physical activity among adults 50 and older: Implications for future physical activity and cognitive functioning research. In: Poon, L., Chodzko-Zajko, W. and Tomporowski, T., Eds., Active Living, Cognitive Functioning, and Aging, Human Kinetics, Champaign.

[9] Lange, B., Flynn, S., \& Rizzo, A. (2009), Game-based telerehabilitation. European Journal of Physical and Rehabilitation Medicine, 45, 143-151.

[10] Basak, C., Boot, W.R., Voss, M.W. and Kramer, A.F. (2008) Can training in a real-time strategy video game attenuate cognitive decline in older adults? Psychology and Aging, 23, 765-777. doi:10.1037/a0013494

[11] Green, C. and Bavelier, D. (2008) Exercising your brain: A review of human brain plasticity and training-induced learning. Psychology and Aging, 23, 692-701. doi:10.1037/a0014345

[12] Van Schaik, P., Blake, J., Pernet, F., Spears, I. and Fencott, C. (2008) Virtual augmented exercise gaming for older adults. CyberPsychology \& Behavior, 11, 103-106. doi:10.1089/cpb.2007.9925

[13] Haddad, J.M., Van Emmerik, R.A., Wheat, J.S. and Hamill, J. (2008) Developmental changes in the dynamical structure of postural sway during a precision fitting task. Experimental Brain Research, 190, 431-441. 


$$
\text { doi:10.1007/s00221-008-1483-9 }
$$

[14] Brandt, J., Spencer, M. and Folstein, M. (1988) The telephone interview for cognitive status. Neuropsychiatry, Neuropsychology, and Behavioral Neurology, 1, 111-117.

[15] Stalvey, B., Owsley, C., Sloane, M.E. and Ball, K. (1999) The life space questionnaire: A measure of the extent of mobility of older adults. Journal of Applied Gerontology, 18, 479-498. doi:10.1177/073346489901800404

[16] Hill, K., Schwarz, J., Kalogeropoulos, A. and Gibson, S. (1996) Fear of falling revisited. Archives of Physical Medicine and Rehabilitation, 77, 1025-1029. doi:10.1016/S0003-9993(96)90063-5

[17] Rose, D. (2010) FallProof!: A comprehensive balance and mobility training program. Human Kinetics, Champaign.

[18] DeVreede, P. (2004) Functional tasks exercise versus resistance exercise to improve daily function in older women: A feasibility study. Archives of Physical Medicine and Rehabilitation, 85, 1952-1961. doi:10.1016/j.apmr.2004.05.006

[19] Dunlap, W., Cortina, J., Vaslow, J. and Burke, M. (1996) Meta-analysis of experiments with matched groups or repeated measures designs. Psychological Methods, 1, 170177. doi:10.1037/1082-989X.1.2.170

[20] Cohen, J. (1992) A power primer. Psychological Bulletin, 112, 155-159. doi:10.1037/0033-2909.112.1.155

[21] Acock, A. (2005) Working with missing values. Journal of Marriage and Family, 67, 1012-1028. doi:10.1111/j.1741-3737.2005.00191.x

[22] Harding, S. and Gardner, A. (2009) Fear of falling. Australian Journal of Advanced Nursing, 27, 94-100. 